\title{
RESTAURAÇÃO INDIRETA DE CERÔMERO: UMA ALTERNATIVA ESTÉTICA VIÁVEL?
}

\author{
Avilmar Passos Galvão Mestre em Odontologia Clínica pela Escola Bahiana \\ de Medicina e Saúde Pública (EBMSP). Professor \\ convidado da EBMSP. \\ Carlos Akio Saback Miura Mestre em Odontologia Clínica pela Escola Bahiana \\ de Medicina e Saúde Pública (EBMSP)' Professor do \\ Curso de Especialização em Odontopediatria da \\ Associação Brasileira de Odontologia - Seção Bahia. \\ Wanessa Maria de Freitas Aras Mestre em Odontologia Clínica pela Escola Bahiana \\ de Medicina e Saúde Pública. Professor da \\ Universidade Estadual do Sudoeste da Bahia.

\begin{abstract}
Resumo
Devido à crescente exigência por estética, têm-se procurado por recursos auxiliares a fim de atender aos anseios e às necessidades dos pacientes. As restaurações metalocerâmicas têm demonstrado sucesso clínico em longo prazo, porém apresentam desvantagem estética em virtude da posterior exposição do metal subjacente. Assim sendo, nas últimas décadas observou-se um intenso desenvolvimento no campo dos biomateriais com o advento das restaurações e próteses metal-free. Dentre estes novos materiais, surgiram os compósitos indiretos com o intuito de minimizar os efeitos da contração de polimerização comuns às resinas compostas. Estes compósitos possibilitaram a melhoria da resistência, a simplicidade na confecção e a redução do custo. Os cerômeros, por sua vez, são resinas compostas reforçadas por partículas cerâmicas e cuja polimerização ocorre fora do ambiente bucal. Por outro lado, ainda permanecem dúvidas quanto à indicação, viabilidade e longevidade destes polímeros. O presente trabalho teve por objetivo analisar as informações clínicas existentes na literatura que suportem a utilização de tais compósitos como materiais restauradores. Conforme o levantamento realizado, concluiu-se que os cerômeros embora representem uma opção restauradora viável, necessitam de mais pesquisas clínicas longitudinais a fim de avaliar o seu comportamento em longo prazo.
\end{abstract}

Palavras-chave: Materiais dentários; Polímeros; Restaurações intra-coronárias; Restaurações extracoronárias.

\section{CEROMER INDIRECT RESTAURATION: A VIABLE AESTHETIC ALTERNATIVE?}

\begin{abstract}
Due to the growing demand for aesthetics, is a search for auxiliary resources in order to meet the aspirations and needs of patients. The metal ceramic restorations have shown clinical success in the long term, but have aesthetic disadvantage because of the exposure of the underlying metal. Thus, in recent decades there was an intense development in the field of biomaterials with the advent of prosthetic restorations and metal-free. Among these new materials, the indirect composite emerged with the aim of minimizing the effects of contraction of polymerization common to the composite resins. These composites allowed for the improvement of strength, the simplicity in the manufacture and reducing cost. The ceromers are resins reinforced by ceramic particles and whose polymerization occurs outside the oral environment. Moreover, doubts remain about the indication, viability and longevity of these polymers. Thus, this study aimed to analyze the information of clinical evidence in the literature that support the use of restorative materials such as composites. Based on the survey conducted, it was concluded that though the polymers represent a viable option restorative, most longitudinal clinical research becomes necessary in order to evaluate their behaviour in the long term.
\end{abstract}

Keywords: Dental materials; Polymers; Inlays; onlays. 


\section{INTRODUÇÃO}

Devido à valorização da estética nos dias atuais, pacientes e profissionais têm procurado por recursos que favoreçam uma aparência dos dentes a mais natural possível. As restaurações indiretas metálicas e metalocerâmicas demonstraram sucesso clínico a longo prazo, porém a visibilidade do metal com o passar do tempo resultou em desvantagens indesejáveis do ponto de vista da cor. O desejo de se alcançar qualidades estéticas mais próximas da estrutura dentária impulsionou os pesquisadores nas últimas décadas para o desenvolvimento de materiais "metal-free" a serem aplicados em coroas e próteses parciais fixas. $^{(1,2)}$

Segundo Kükrer et al., ${ }^{(3)}$ além da exigência estética, outros aspectos dos materiais restauradores fizeram com que houvesse a preocupação em tentar recursos alternativos. A alegada toxicidade do amálgama de prata e a contração de polimerização das resinas compostas diretas, gerando stress na interface adesiva são alguns dos exemplos. Além disso, apesar das cerâmicas possuírem excelentes propriedades estéticas e biocompatibilidade, quando usadas em inlays/onlays têm como principal desvantagem o risco à fratura em virtude da sua natureza friável frente ao stress mastigatório e/ou oclusal. ${ }^{(4,5,6)}$

Pesquisas direcionadas ao desenvolvimento de materiais menos friáveis têm gerado alternativas interessantes às restaurações cerâmicas. Uma vantagem que chama a atenção para com os compósitos, citada por Gemalmaz e Kükrer ${ }^{(7)}$ e Ohlmann et al., ${ }^{(2)}$ é a simplicidade da técnica na medida em que é possível eliminar os procedimentos de fundição e confecção de gesso refratário, além de realizar reparos caso sejam necessários. Ademais, o custo torna-se mais acessível e amplia-se a gama de recursos estéticos, visando atender dentro dos limites científicos e clínicos aos anseios dos pacientes.

Os compósitos resinosos, em alguns casos, têm sido o material de escolha quando da indicação de restaurações indiretas do tipo inlays/ onlays. Com o objetivo de melhorar a resistência e a estética, a segunda geração dos compósitos, conhecida como cerômeros foi introduzida no mercado odontológico. ${ }^{(3,7)}$ Tais "cerômeros" são confeccionados em laboratório e polimerizados por meio da luz, calor e/ou vácuo. ${ }^{(7)}$

Os cerômeros, por serem confeccionados fora da boca, minimizam os problemas da contração de polimerização e insuficiente adaptação marginal das resinas compostas diretas além de não possuir a friabilidade das estruturas cerâmicas. ${ }^{(2,3,7,8)}$ Entretanto, ainda há o 
questionamento e por vezes, o receio por parte dos profissionais em utilizar estes polímeros na sua prática diária. É possível que os cerômeros sejam uma alternativa estética frente às resinas compostas diretas e às cerâmicas? Para responder a esta questão e outras, é primordial a execução de trabalhos, principalmente, clínicos que analisem as avaliações in vivo do comportamento dos novos compósitos, permitindo assim, delinear as possíveis falhas e causas bem como a previsibilidade e o sucesso no tratamento estético.

Portanto, o objetivo deste trabalho foi analisar as evidências clínicas existentes na literatura a respeito, entre outros aspectos, da indicação, viabilidade e longevidade dos polímeros na reabilitação protética.

\section{METODOLOGIA}

Inicialmente foi realizado um levantamento bibliográfico na Biblioteca Interinstitucional Eurydice Pires de Sant'anna localizada no Centro de Pesquisas Gonçalo Muniz, em Brotas, Salvador. Nesta instituição, obteve-se acesso livre ao site www.ncbi.nlm.nih.gov/PubMed, o qual dispõe de banco de dados do Medline, sendo as palavras-chave utilizadas para a consulta ceromer, inlays/onlays, clinical performance e polymers. Os critérios de inclusão foram estudos clínicos randomizados ou nãorandomizados, redigidos em inglês e publicados de 1998 a 2007. Os artigos eram excluídos pelo título ou pelo abstract. Após a exclusão, foram selecionados nove trabalhos, e posteriormente, lidos na íntegra. Nova pesquisa foi desenvolvida por meio da fonte de dados do Google Scholar e três artigos foram então acrescentados ao levantamento.

\section{RESULTADOS}

Dos artigos selecionados, três eram estudos do tipo retrospectivo e oito eram caracterizados como prospectivos. Um trabalho realizado tanto em in vitro quanto em in vivo foi adicionado aos demais para análise. Todos os estudos clínicos referentes aos cerômeros encontram-se resumidos e dispostos na Tabela 1. 
Tabela 1 - Detalhes dos estudos clínicos dispostos por ordem cronológica

\begin{tabular}{|c|c|c|c|c|c|}
\hline $\begin{array}{l}\text { ESTUDO } \\
\text { CLÍNICO }\end{array}$ & $\begin{array}{c}\text { ACOMPA- } \\
\text { NHAMEN- } \\
\text { TO }\end{array}$ & $\begin{array}{c}\text { AMOSTRA } \\
\text { PACIENTES/ } \\
\text { RESTAURAÇÕES }\end{array}$ & $\begin{array}{c}\text { RESTAURAÇÃO/ } \\
\text { SISTEMA }\end{array}$ & CRITÉRIOS & RESULTADOS \\
\hline $\begin{array}{l}\text { Koch \& } \\
\text { García- } \\
\text { Godoy } \\
(2000)\end{array}$ & $\begin{array}{c}2 \text { a } 5 \text { anos } \\
\text { (cada } 6 \\
\text { meses) }\end{array}$ & $12 / 41$ & $\begin{array}{c}\text { Coroas ouro fundido/ } \\
\text { Degulor M, } \\
\text { Compósito/ Artglass e } \\
\text { Cerâmica/ Empress }\end{array}$ & $\begin{array}{c}\text { USPHS } \\
\text { modificado }\end{array}$ & $\begin{array}{l}\text { Coroas } \\
\text { laboratoriais podem } \\
\text { ser usadas em } \\
\text { molares jovens } \\
\text { permanentes com } \\
\text { defeitos de } \\
\text { desenvolvimento. }\end{array}$ \\
\hline $\begin{array}{l}\text { Monaco et } \\
\text { al. (2001) }\end{array}$ & $\begin{array}{c}6,12 \text { e } 18 \\
\text { meses }\end{array}$ & $25 / 43$ & Inlay e Onlay / Targis & USPHS* & $\begin{array}{l}\text { Bom serviço } \\
\text { clínico. }\end{array}$ \\
\hline $\begin{array}{l}\text { Redman et } \\
\text { al. (2003) }\end{array}$ & $\begin{array}{c}5 \text { meses a } 6 \\
\text { anos }\end{array}$ & $31 / 225$ & $\begin{array}{c}\text { Microparticulada } \\
\text { (Durafill); } \\
\text { Microhíbrida } \\
\text { (Herculite: técnica } \\
\text { direta e indireta); } \\
\text { Cerômero indireto } \\
\text { (Artglass) }\end{array}$ & $\begin{array}{c}\text { USPHS } \\
\text { modificado }\end{array}$ & $\begin{array}{l}\text { Bom tempo de } \\
\text { sobrevida de curto } \\
\text { a médio prazo. }\end{array}$ \\
\hline $\begin{array}{l}\text { Yilmaz \& } \\
\text { Gemalmaz } \\
(2003)\end{array}$ & $\begin{array}{c}\text { Baseline, } 6 \text { e } \\
12 \text { meses }\end{array}$ & $26 / 40$ & Inlay/ Targis & $\begin{array}{c}\text { USPHS } \\
\text { modificado }\end{array}$ & $\begin{array}{l}\text { Escores clínicos } \\
\text { aceitáveis. }\end{array}$ \\
\hline $\begin{array}{l}\text { Kürker } e t \\
\text { al. (2004) }\end{array}$ & 53 meses & $51 / 99$ & Inlay/Targis & $\begin{array}{c}\text { USPHS } \\
\text { modificado }\end{array}$ & $\begin{array}{l}\text { Alto nível de } \\
\text { aceitação do } \\
\text { paciente. } \\
\text { Baixa taxa de } \\
\text { fratura e baixo } \\
\text { índice de cárie } \\
\text { secundária. }\end{array}$ \\
\hline $\begin{array}{l}\text { Rammelsber } \\
\text { g et al. } \\
(2005)\end{array}$ & $\begin{array}{c}1 \text { mês e } \\
1,2,3 \text { anos }\end{array}$ & $71 / 114$ & Coroas/ Artglass & $\begin{array}{l}\text { VAS (visual } \\
\text { rating scales) }\end{array}$ & $\begin{array}{l}\text { Índice de sobrevida } \\
\text { de } 96 \% \text {. }\end{array}$ \\
\hline $\begin{array}{l}\text { Gemalmaz } \\
\& \text { Kürker } \\
\text { (2006) }\end{array}$ & Não & $20 / 20$ & Inlay/ Targis & Não & $\begin{array}{l}\text { Foi avaliada a } \\
\text { adaptação marginal } \\
\text { e não a } \\
\text { performance } \\
\text { clínica. }\end{array}$ \\
\hline
\end{tabular}




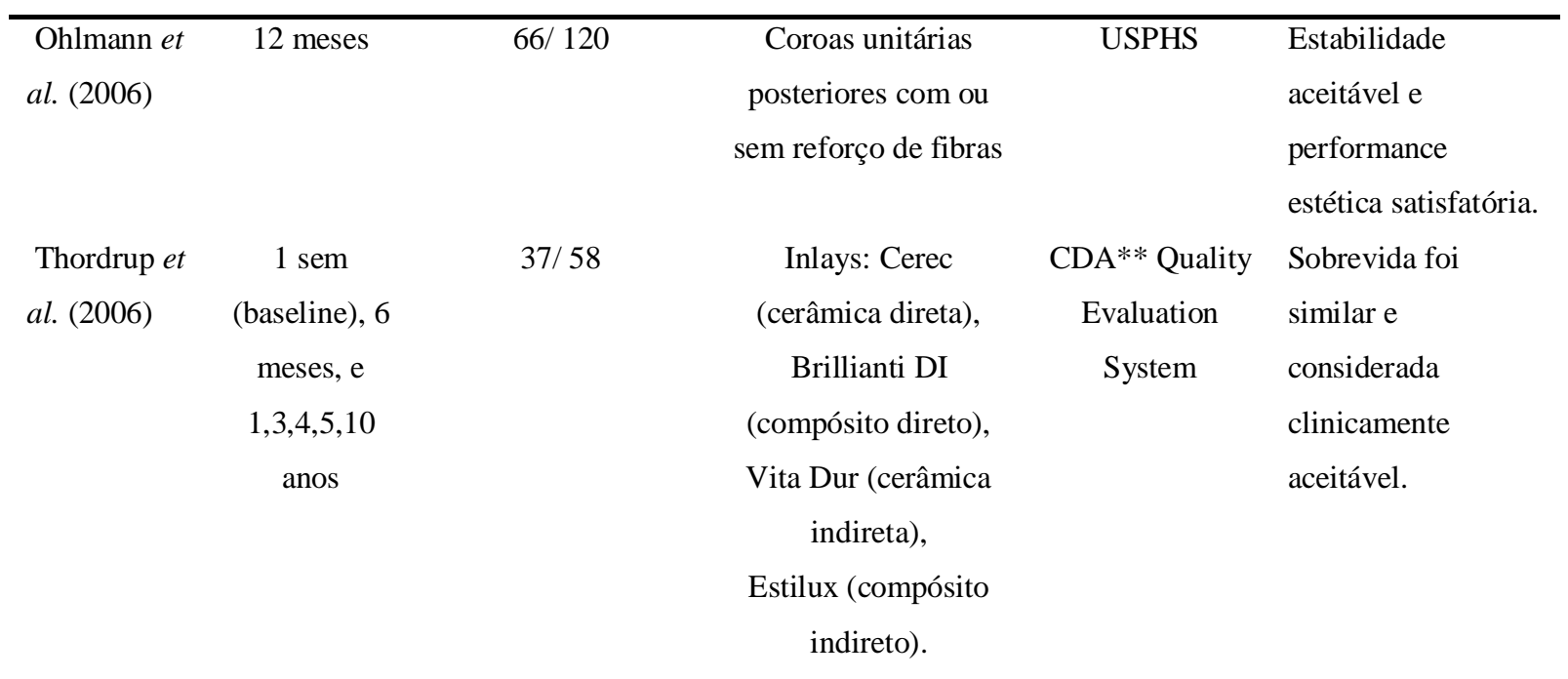

* United States Public Health Service Criteria

** California Dental Association

\section{DISCUSSÃO}

A princípio foi constatada, durante o levantamento bibliográfico, a ausência de concordância em relação à denominação "cerômeros". Outras expressões correlacionadas foram encontradas tais como, "resinas laboratoriais", "resinas compostas indiretas", "polímeros", "ormocer" e "compósitos indiretos". Tal fato dificulta sobremaneira a pesquisa e ao mesmo tempo podem gerar dúvidas ou interpretações errôneas a despeito deste material.

Os estudos clínicos selecionados neste trabalho embora quantitativamente limitados fornecem informações relevantes quanto à utilização e às propriedades mecânicas dos cerômeros. No que diz respeito às indicações dos cerômeros, Freilich et al. ${ }^{(2)}$ citam situações onde a área a ser reabilitada requer um excelente resultado estético e há o desejo expresso pelo paciente em ter uma prótese cuja cor seja similar aos dentes naturais. Ademais, também podem ser aplicados se o espaço para o antagonista for pequeno, caso seja necessária uma retenção adicional e para pontes fixas curtas. Koch e García-Godoy ${ }^{(9)}$ defendem o uso de coroas de cerômero fabricadas laboratorialmente em molares permanentes jovens com defeitos de esmalte, como opção às coroas metálicas.

Ohlmann et al. ${ }^{(2)}$ alegam que o primeiro requisito necessário à escolha das coroas de polímeros, como tratamento alternativo às metalocerâmicas convencionais, é serem aquelas resistentes às forças mastigatórias. Estes autores ao realizarem um estudo de 1 ano de acompanhamento verificaram que coroas unitárias de polímeros com e sem fibra de vidro em 
dentes posteriores não revelaram influência clinicamente relevante da presença de material de reforço para a estabilidade das restaurações. Os resultados mostraram estabilidade e desempenho estético aceitáveis, entretanto deve-se considerar que tal avaliação foi realizada em um período de apenas 12 meses.

Estudos como o de Kükrer et al., ${ }^{(3)}$ Rammelsberg et al. ${ }^{(6)}$ e Monaco et al. ${ }^{(10)}$ observaram a resistência à fratura destes compósitos. Rammelsberg et al. ${ }^{(6)}$ utilizaram uma amostra de 100 coroas do Sistema Artglass com período de acompanhamento de 3 anos e constataram a ocorrência de apenas 6 fraturas. Com relação à taxa de sobrevida destas restaurações, verificou-se um índice de $96 \%$, quase similar às coroas de cerâmicas. O desempenho das inlays/onlays de cerômero do Sistema Targis foi considerado bom após um período de 18 meses. ${ }^{(10)}$ Para fraturas, ao fim de 27,6 meses o índice foi de $1 \%$ no estudo de Kükrer et al. ${ }^{(3)}$ concordando com os dados encontrados por Monaco et al. ${ }^{(10)}$ Isto implica, conforme os achados de Kükrer et al., ${ }^{(3)}$ que o uso de compósitos da nova geração e menos friáveis do que as cerâmicas reduz o risco de fratura em restaurações de inlay e onlay. É importante salientar a verificação feita por Felden et al. ${ }^{(4)}$ e Krämer et al. ${ }^{(5)}$ na qual a fratura de corpo constitui a principal causa de falha das cerâmicas.

Thordrup et al. ${ }^{(11)}$ em uma pesquisa clínica prospectiva de 10 anos, avaliaram 4 tipos de inlays diretas e indiretas de cerâmica e compósito. De um total de 58 restaurações, 6 inlays (3 de cerâmica, 1 de compósito indireto e 2 de compósito direto) sofreram reparos devido a pequenas fraturas. E os índices de sucesso, incluindo as restaurações com reparos, chegaram a $80 \%$. Não houve diferença estatisticamente significante com relação à sobrevivência dos diferentes tipos de restaurações, e os resultados foram clinicamente aceitáveis. Além disso, houve decréscimo similar na qualidade estética para as diferentes inlays. Porém, observa-se que 9 inlays (sendo 6 de cerâmica e 3 de compósito direto) necessitaram ser substituídas devido às cáries secundárias ou fraturas. Por outro lado, 3 unidades restauradas com cerômeros apresentaram hipersensibilidade persistente ou dano pulpar. Os autores não apresentaram justificativas para este fato e uma das hipóteses pode estar relacionada à cimentação adesiva. Kükrer et al. ${ }^{(3)}$ observaram a longevidade clínica promissora dos compósitos indiretos com pequenos índices de fratura e recidiva de cárie. Koch e GarcíaGodoy $^{(9)}$ afirmam que pacientes com alto risco à cárie poderiam ser beneficiados com o uso de cerômeros no tratamento restaurador.

A deterioração da adaptação marginal é um problema comum para as restaurações adesivas, tanto de compósitos quanto das cerâmicas. ${ }^{(7,8)}$ As restaurações indiretas do tipo inlay 
e onlay oferecem um excelente controle da margem proximal e do ponto de contato, pois durante a confecção de tais materiais, os mesmos sofrem contração de polimerização em ambiente fora da boca, e por isso, a etapa da cimentação transcorre livre de maiores tensões. Isto minimiza os efeitos negativos do stress causado pela contração de polimerização e problemas de adaptação marginal, frequentemente verificados em restaurações diretas posteriores com resina composta. ${ }^{(7,10)}$ Dessa maneira, Gemalmaz e Kükrer ${ }^{(7)}$ destacam que os cerômeros representam uma alternativa viável às restaurações diretas. Monaco et al. ${ }^{(10)}$ utilizaram 23 onlays e 20 inlays, colocadas em 25 pacientes, e concluíram que as mesmas demonstraram excelente integridade marginal e não sofreram deformação ou perda estrutural.

Um critério adicional para o sucesso clínico das coroas de cerômeros consiste na ausência de falhas. Rammelsberg et al. ${ }^{(6)}$ e Ohlmann et al. ${ }^{(2)}$ citam o termo delamination para se referirem à complicação mais comum em coroas de compósitos reforçadas por fibras. Fenômeno este que está relacionado ao mecanismo de adesão entre a matriz resinosa e as fibras de vidro. Quando ocorre a contaminação por água, a tensão superficial entre as fibras de vidro e o polímero tende a diminuir, e consequentemente, o índice de descamação tende a aumentar. Também, foi relatado o maior acúmulo de placa nos polímeros reforçados, em virtude da porosidade e eventual exposição das fibras. Entretanto, estes efeitos não comprometem o tratamento em si, pois os próprios autores afirmam que esses problemas podem ser contornados através da polimerização do material sob pressão e calor. Quanto à adesão da placa sobre os polímeros, além do tratamento térmico e bárico que promovem a redução das irregularidades superficiais, ${ }^{(2)}$ uma instrução de higiene oral aos pacientes se faz necessária.

É pertinente a colocação de Ohlmann et al. ${ }^{(2)}$ ao ressaltarem que paralelo à análise da taxa de sobrevida das restaurações, a aparência estética exerce um papel importante na avaliação do sucesso clínico. Esta observação reveste-se de importância quando os pacientes são indagados a respeito do tratamento. A boa aceitação dos mesmos em relação ao resultado estético dos compósitos foi registrada em alguns artigos. ${ }^{(6,11,12)}$ Vale ressaltar que Thordrup et al. ${ }^{(11)}$ avaliaram 4 diferentes tipos de inlays estéticas, e todos os pacientes mostraram-se satisfeitos com o procedimento restaurador. Apesar de ter ocorrido degradação marginal e alteração na textura em algumas inlays/onlays de cerômero do Sistema Targis no seu trabalho, Kükrer et al. ${ }^{(3)}$ concluíram que houve um grande nível de aprovação dos pacientes para as restaurações, em um período de 27,6 meses de acompanhamento clínico. E até mesmo, no tratamento odontopediátrico, os pais têm preferido as restaurações estéticas às metálicas. ${ }^{(9)}$ 
A análise dos trabalhos revela falta de consenso no que se refere à indicação de polímeros em pacientes portadores de bruxismo e ou apertamento dentário. No estudo de Thordrup et al. ${ }^{(11)}$ no qual se utilizou cerômero e cerâmica, pacientes com hábitos parafuncionais foram excluídos. Kükrer et al. ${ }^{(3)}$ intencionalmente colocaram $29 \%$ das restaurações indiretas de compósitos em pacientes que apresentavam hábitos parafuncionais. Houve o cuidado de cimentar as inlays de forma a não interferir na oclusão. Os autores observaram baixo índice de fratura e verificaram que as inlays do Sistema Targis funcionaram satisfatoriamente dentro do período médio de 28 meses. Freilich et al. ${ }^{(1)}$ e Redman et al. ${ }^{(12)}$ consideraram válida a exclusão destes pacientes como candidatos às restaurações indiretas de compósitos, ao afirmarem que os polímeros podem ser indicados caso o indivíduo não apresente um quadro parafuncional ou situações de sobrecarga. Rammelsberg et al. $^{(6)}$ fornecem um dado esclarecedor a essa questão. Estes pesquisadores observaram que coroas de polímeros cimentadas em dentes posteriores não mostraram perda de suporte oclusal em oclusão cêntrica dentro de 3 anos e o desempenho clínico foi considerado aceitável pelos pacientes. De uma maneira geral, conforme o tipo de restauração haverá uma menor ou maior exigência em relação ao stress mastigatório. Entretanto, estudos adicionais com tamanho amostral mais representativo e tempo de avaliação satisfatório são requeridos.

Por sua vez, as próteses parciais fixas extra-coronárias e intra-coronárias polimerizadas a luz e calor feitas com uma matriz de compósitos reforçada por fibra unidirecional e pré-impregnada (Fibrekor) e revestida com um compósito particulado híbrido (Sculpture) apresentaram bons resultados clínicos durante quatro ou mais anos, conforme trabalho desenvolvido por Freilich et al. ${ }^{(1)}$ Estes dados refletem que o sucesso clínico estava primariamente relacionado ao desenho da infra-estrutura e ao reforço com fibra de vidro.

Redman et al. ${ }^{(12)}$ defendem a aplicabilidade dos compósitos indiretos à base de resina tais como os do Sistema Artglass no tratamento de desgaste dentário anterior localizado, considerando-os como a primeira escolha a curto e médio prazo. Segundo os autores, as falhas tais como fratura de corpo, rugosidade superficial e descoloração da restauração são incomuns para estes materiais.

De acordo com Koch e García-Godoy, ${ }^{(9)}$ o uso de coroas laboratoriais tradicionalmente não tem sido recomendado para crianças em decorrência do custo e da dificuldade no acabamento das margens. Esta contra-indicação é relativa, pois em se tratando de custobenefício, as coroas de aço são relativamente baratas. Porém, em pacientes odontopediátricos alérgicos ao níquel e ao cromo, tal utilização deve ser evitada. Por outro lado, como o molar 
permanente jovem encontra-se em processo de erupção, as margens permanecerão supragengivais, possibilitando o acabamento e polimento das mesmas. Além disso, conforme Koch e García-Godoy, ${ }^{(9)}$ há o risco de exposição pulpar durante o preparo, contudo não há estudos que demonstrem ser um problema de relevância clínica.

Um material para ser considerado bom deve estar corretamente indicado. Assim sendo, os cerômeros podem ser a alternativa estética conforme as expectativas do paciente, associada às particularidades do caso e à experiência do profissional. Certamente, mais estudos clínicos são requeridos com a finalidade de consolidar o conhecimento acerca dos compósitos. Portanto, as evidências suportadas pela literatura consultada, apesar das limitações, indicam a viabilidade e a longevidade de curto em médio prazo para as restaurações estéticas de resinas compostas indiretas na reabilitação oral.

\section{CONCLUSÕES}

Com base nos estudos clínicos analisados, pode-se inferir que:

1. As resinas compostas indiretas representam uma opção restauradora estética viável se respeitadas as indicações além dos anseios e necessidades dos pacientes;

2. E pesquisas clínicas longitudinais se fazem necessárias a fim de avaliar o comportamento a longo prazo destas restaurações.

\section{REFERÊNCIAS}

1. Freilich MA, Meiers JC, Duncan JP, Eckrote KA, Goldberg AJ. Clinical evaluation of fiber-reinforced fixed bridges. JADA 2002; 133: 1524-34.

2. Ohlmann B, Dreyhaupt J, Schmitter M, Gabbers O, Hassel A, Rammelsberg P. Clinical performance of posterior metal-free polymer crowns with and without fiber reinforcement. One-Year Results of a Randomized Clinical Trial. J Dent 2006; 34: 757-62.

3. Kükrer D, Gemalmaz D, Kuybulu EO, Bozkurt FO. A Prospective clinical study of ceromer inlays: results up to 53 months. Int J Prosthodont 2004; 17: 17-23.

4. Felden A, Schmalz G, Federlin M, Hiller KA. Retrospective clinical investigation and survival analysis on ceramic inlays and partial ceramic crowns: results up to 7 years. Clin Oral Investig 1998; 2: 161- 67. 
5. Krämer N, Frankenberg R, Pelka M, Petschelt A. IPS Empress inlays and onlays after four years - a clinical study. J Dent 1999; 27: 325-31.

6. Rammelsberg P, Spiegl K, Eickemeyer G, Schmitter M. Clinical performance of metal-free polymer crowns after 3 years in service. J Dent 2005; 33: 517-23.

7. Gemalmaz D, Kükrer D. In Vivo and In Vitro evaluation of marginal fit of class II ceromer inlays. J Oral Rehabil 2006; 33: 436-42.

8. Yilmaz D, Gelmamaz D. Clinical evaluation of class II targis inlays: preliminary results After 1 Year. J Oral Rehabil 2003; 30: 855-60.

9. Koch MJ, García-Godoy F. The Clinical performance of laboratory-fabricated crowns: Placed on First Permanent Molars With Developmental Defects. JADA 2000; 131: 1285-90.

10. Monaco C, Baldissara P, Dall'Orologio GD, Scotti R. Short-Term clinical evaluation of inlay and onlay restorations made with a ceromer. Int J Prosthodont 2001; 14: 81-86.

11. Thordrup M, Isidor F, Hörsted-Bindslev P. A Prospective clinical study of indirect and direct composite and ceramic inlays: ten-year results. Quintessence Int 2006; 37: 139-44.

12. Redman CDJ, Hemmings KW, Good JA. The survival and clinical performance of resinbased composite restorations used to treat localised anterior tooth wear. Brit Dent J 2003; 194 (10): 566-72. 